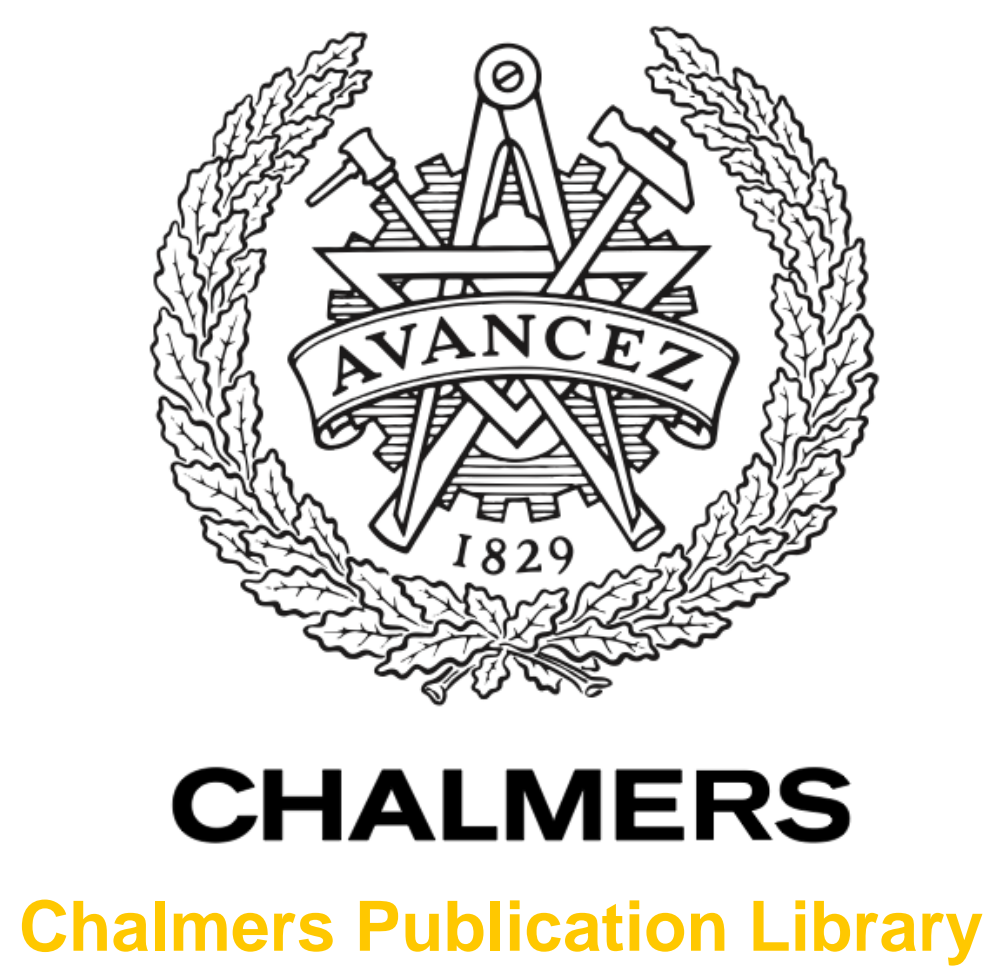

Influence of Different Sources of Noise on Epileptic Spike EEG Source Localization

This document has been downloaded from Chalmers Publication Library (CPL). It is the author's version of a work that was accepted for publication in:

Proceedings of SPIE - Medical Imaging 2013: Biomedical Applications in Molecular, Structural, and Functional Imaging,

Citation for the published paper:

Shirvany, Y. ; Chen, X. ; Dhanpalwar, P. (2013) "Influence of Different Sources of Noise on Epileptic Spike EEG Source Localization". Proceedings of SPIE - Medical Imaging 2013: Biomedical Applications in Molecular, Structural, and Functional Imaging,, vol. 8672

http://dx.doi.org/10.1117/12.2007118

Downloaded from: http://publications.lib.chalmers.se/publication/172770

Notice: Changes introduced as a result of publishing processes such as copy-editing and formatting may not be reflected in this document. For a definitive version of this work, please refer to the published source. Please note that access to the published version might require a subscription. 


\title{
Influence of Different Sources of Noise on Epileptic Spike EEG Source Localization
}

\author{
Yazdan Shirvany ${ }^{a d}$, Xinyuan Chen $^{a}$, Prathamesh Sharad Dhanpalwar ${ }^{a}$, \\ Mahdieh Mir Hashemi ${ }^{c}$, Fredrik Edelvik ${ }^{b}$, Mikael Persson $^{a d}$ \\ ${ }^{a}$ Dept. of Signals and Systems, Chalmers University of Technology, Gothenburg, Sweden; \\ ${ }^{b}$ Fraunhofer-Chalmers Research Centre, Chalmers Science Park, Gothenburg, Sweden; \\ ${ }^{c}$ Dept. of Computer Science, Chalmers University of Technology, Gothenburg, Sweden; \\ ${ }^{d}$ MedTech West, Sahlgrenska University Hospital, Gothenburg, Sweden;
}

\begin{abstract}
Spike EEG source localization results are influenced by different errors and approximations, e.g., head-model complexity, EEG signal noise, electrode misplacements, tissue anisotropy, tissue conductivity noise as well as numerical errors. For accurate source localization, understanding the affects of these errors on the source localization is very crucial. Six finite element head models are selected for a head-model complexity study. A reference head model is used to create the synthetic EEG signals by placing a dipole inside the model to mimic the epileptic spike activity. To understand the influence of EEG signal noise, tissue conductivity noise and electrode misplacements on the EEG source localization, different level of noises are added to EEG signals, tissue conductivities and electrode positions, independently. To investigate the influence of white matter anisotropy, a realistic head model generated from T1-weighted MRI is used and the conductivity anisotropy for the white matter is calculated from diffusion tensor imaging (DTI). Major findings of the study include (1) the CSF layer plays an important role to achieve an accurate source localization result, $(2)$ the source localization is very sensitive to the tissue conductivity noises, (3) one centimeter electrode misplacement cause approximately $8 \mathrm{~mm}$ localization error, (4) the source localization is robust with respect to the EEG signal noise and (5) the model with white matter anisotropy has small source localization error but large amplitude and orientation errors compared to the isotropic head model.
\end{abstract}

Keywords: EEG Source Localization, Model Complexity, EEG noise, Conductivity Noise, Electrode Misplacements, White Matter Anisotropy

\section{INTRODUCTION}

Surgical therapy has become an important therapeutic alternative for patients with medically intractable epilepsy. Correct and anatomically precise localization of the epileptic focus is mandatory to decide if resection of brain tissue is possible. The most important diagnosis tool used at epilepsy surgery centers is electroencephalography (EEG), which is used to find the source of activities inside the brain by measuring the voltage potential on the scalp with the EEG electrodes at different locations. The brain activity is often modeled as a current dipole. It is shown ${ }^{1}$ that this current dipole is an acceptable approximation for modeling the neural activities in the brain. The procedure of the EEG source localization deals with two problems. First, the forward problem to find the scalp potentials for the given current dipole(s) inside the brain and second the inverse problem to estimate the source(s) that fits with the given potential distribution at the scalp electrodes. Thus, source localization requires an accurate solution of the inverse problem with a realistic computational effort for solving the forward problem.

Source localization is heavily dependent on the choice of dipole model and several different alternatives have been suggested in the literature, ${ }^{2,3}$ and its accuracy is affected by different factors including, head-modeling complexity, EEG signal noise, tissue conductivity noise and electrode misplacements. Moreover, the brain white matter has an anisotropic conductivity with a ratio of about 1:9 (normal:parallel to fibers). ${ }^{11}$ No direct technique

Further author information: (Send correspondence to Y. Shirvany)

Y. Shirvany: (ه) y_shirvany@yahoo.com, yazdan.shirvany@chalmers.se

Medical Imaging 2013: Biomedical Applications in Molecular, Structural, and Functional Imaging,

edited by John B. Weaver, Robert C. Molthen, Proc. of SPIE Vol. 8672, 86720N

(C) 2013 SPIE · CCC code: $1605-7422 / 13 / \$ 18 \cdot$ doi: 10.1117/12.2007118

Proc. of SPIE Vol. 867286720 N-1 


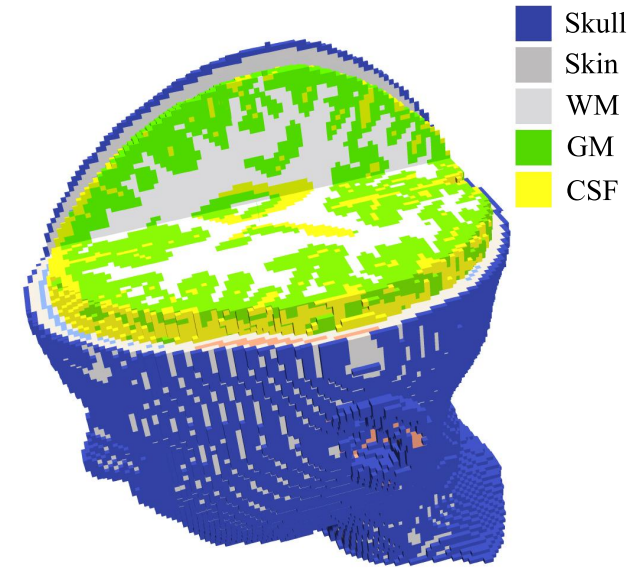

(a)

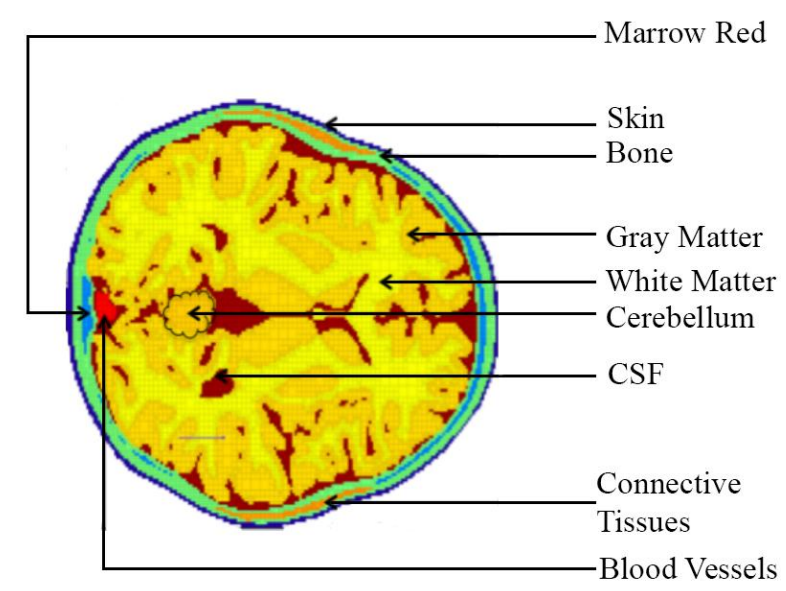

(b)

Figure 1: a) The 3D head model and b) the 2D reference head model with the label of each tissue.

exists for robust and non-invasive measurement of the conductivity properties, however, recently the relation between the effective electrical conductivity tensor of brain tissue and the effective water diffusion tensor, as measured by Diffusion Tensor MRI (DT-MRI), was formulated. ${ }^{13,14}$ Understanding the influence of these errors is very important to have a reliable source localization for pre-surgical workup. EEG-based source localization is an active field of research, ${ }^{4}$ but partly due to the aforementioned shortcomings the computational techniques are not yet part of the standard pre-surgical diagnostic workup.

The paper is outlined as follows. In Section 2 the influence of head model complexity, EEG signal noise, conductivity noise and electrode misplacement on the EEG source localization are investigated. Moreover, in Section 2, two methods, i.e., direct and volume normalized, for extracting the white matter anisotropy form DT-MRI is presented. In Section 3, the numerical results are presented. Finally, Section 4 summarizes the paper and conclusions are drawn.

\section{METHODS}

Here we use the modified subtraction method ${ }^{5}$ to model the dipole in the forward problem. The inverse problem in EEG source localization uses the scalp EEG signal to estimate the corresponding current source inside the brain and it is an underdetermined problem and no unique solution can usually be given. To attain uniqueness it is necessary to impose a priori knowledge on the source distribution. For the inverse problem here we follow the common practice and choose the parameters such that we have the best fit in least squares sense. We do an exhaustive search pattern, i.e., inversion is performed for each possible source location in the cortex area inside the brain, and the location producing the smallest residual norm is selected as the best possible source location.

\subsection{Head-Model Complexity}

The aim of this section is to understand the effects of head-model complexity on the EEG source localization and find out the optimal number of tissues for the head-model. Six head models with 4 to 9 tissues were compared with a reference head model, including all available tissues, see Fig. 1b. 
Table 1: Tissue names and conductivity values in each head model.

\begin{tabular}{|c|c|c|c|c|c|c|c|c|c|c|c|}
\hline & & GM & WM & CSF & Fat & $\begin{array}{c}\text { Bone } \\
\text { Marrow } \\
\end{array}$ & Skin & Skull & $\begin{array}{l}\text { Connective } \\
\text { Tissue }\end{array}$ & Cerebellum & $\begin{array}{l}\text { Blood } \\
\text { Vessel } \\
\end{array}$ \\
\hline \multirow{2}{*}{ Ref. Mode } & \multirow{2}{*}{$\begin{array}{l}\text { value } \\
\mathrm{Y} / \mathrm{N} \\
\end{array}$} & 0.089 & 0.0581 & 2.000 & 0.0208 & 0.0018 & 0.0002 & 0.0201 & 0.1628 & 0.109 & 0.7 \\
\hline & & $\mathrm{Y}$ & $\mathrm{Y}$ & $\mathrm{Y}$ & $\mathrm{Y}$ & $\mathrm{Y}$ & $\mathrm{Y}$ & $\mathrm{Y}$ & $\mathrm{Y}$ & $\mathrm{Y}$ & $\mathrm{Y}$ \\
\hline \multirow{2}{*}{ Model \#1 } & \multirow{2}{*}{$\begin{array}{l}\text { value } \\
\mathrm{Y} / \mathrm{N}\end{array}$} & 0.0581 & 0.0581 & 2.000 & 0.0208 & 0.0018 & 0.0002 & 0.0201 & 0.1628 & 0.109 & 0.7 \\
\hline & & $\mathrm{N}$ & $\mathrm{Y}$ & $\mathrm{Y}$ & $\mathrm{Y}$ & $\mathrm{Y}$ & $\mathrm{Y}$ & $\mathrm{Y}$ & $\mathrm{Y}$ & $\mathrm{Y}$ & $\mathrm{Y}$ \\
\hline \multirow{2}{*}{ Model \#2 } & \multirow{2}{*}{$\begin{array}{l}\text { value } \\
\mathrm{Y} / \mathrm{N}\end{array}$} & 0.0581 & 0.0581 & 0.0581 & 0.0208 & 0.0018 & 0.0002 & 0.0201 & 0.1628 & 0.109 & 0.7 \\
\hline & & $\mathrm{N}$ & $\mathrm{Y}$ & $\mathrm{N}$ & $\mathrm{Y}$ & $\mathrm{Y}$ & $\mathrm{Y}$ & $\mathrm{Y}$ & $\mathrm{Y}$ & $\mathrm{Y}$ & $\mathrm{Y}$ \\
\hline \multirow{2}{*}{ Model \#3 } & \multirow{2}{*}{$\begin{array}{l}\text { value } \\
\mathrm{Y} / \mathrm{N}\end{array}$} & 0.089 & 0.0581 & 2.000 & 0.0201 & 0.0201 & 0.0002 & 0.0201 & 0.089 & 0.089 & 2.000 \\
\hline & & $\mathrm{Y}$ & $\mathrm{Y}$ & $\mathrm{Y}$ & $\mathrm{N}$ & $\mathrm{N}$ & $\mathrm{Y}$ & $\mathrm{Y}$ & $\mathrm{N}$ & $\mathrm{N}$ & $\mathrm{N}$ \\
\hline \multirow{2}{*}{ Model \#4 } & \multirow{2}{*}{$\begin{array}{l}\text { value } \\
\mathrm{Y} / \mathrm{N}\end{array}$} & 0.089 & 0.0581 & 0.089 & 0.0201 & 0.0201 & 0.0002 & 0.0201 & 0.089 & 0.089 & 2.000 \\
\hline & & $\mathrm{Y}$ & $\mathrm{Y}$ & $\mathrm{N}$ & $\mathrm{N}$ & $\mathrm{N}$ & $\mathrm{Y}$ & $\mathrm{Y}$ & $\mathrm{N}$ & $\mathrm{N}$ & $\mathrm{N}$ \\
\hline \multirow{2}{*}{ Model \#5 } & \multirow{2}{*}{$\begin{array}{l}\text { value } \\
\mathrm{Y} / \mathrm{N} \\
\end{array}$} & 0.089 & 0.0581 & 0.0581 & 0.0201 & 0.0201 & 0.0002 & 0.0201 & 0.089 & 0.089 & 2.000 \\
\hline & & $\mathrm{Y}$ & $\mathrm{Y}$ & $\mathrm{N}$ & $\mathrm{N}$ & $\mathrm{N}$ & $\mathrm{Y}$ & $\mathrm{Y}$ & $\mathrm{N}$ & $\mathrm{N}$ & $\mathrm{N}$ \\
\hline \multirow{2}{*}{ Model \#6 } & \multirow{2}{*}{$\begin{array}{l}\text { value } \\
\mathrm{Y} / \mathrm{N}\end{array}$} & 0.089 & 0.0581 & 0.0201 & 0.0201 & 0.0201 & 0.0002 & 0.0201 & 0.089 & 0.089 & 2.000 \\
\hline & & $\mathrm{Y}$ & $\mathrm{Y}$ & $\mathrm{N}$ & $\mathrm{N}$ & $\mathrm{N}$ & $\mathrm{Y}$ & $\mathrm{Y}$ & $\mathrm{N}$ & $\mathrm{N}$ & $\mathrm{N}$ \\
\hline
\end{tabular}

Table 1 shows all the head models with their tissue names and conductivities values. In Table 1 , the $\mathrm{Y}$ " in each cell means that the corresponding tissue is present in the model and " $\mathrm{N}$ " means the tissue is not included in the model. A reference head model was used to create synthetic EEG signals by placing an artificial dipole source inside the model to mimic the brain activity.

\subsection{Noise Study}

A more realistic case closer to the clinical practice is to add noise to the model. To understand the influence of noise on the EEG source localization here different levels of noise are added to $a$ ) EEG signals, $b$ ) tissue conductivities and $c$ ) electrode positions. Model \#3 that obtained best results without noise (see Section 3.1) is used. In the real EEG, signal to noise ratio (SNR) which is defined as

$$
S N R=10 \log \left(\frac{P_{\text {signal }}}{P_{\text {noise }}}\right),
$$

usually has a value between 6 and $10 \mathrm{~dB}^{6}$ where $P_{\text {signal }}$ and $P_{\text {noise }}$ are the power of the signal and the power of the noise, respectively. The additive noise is generated such that its power spectrum matches the power spectrum of human EEG. ${ }^{7}$

For the conductivity noise a random Gaussian noise with variance $\delta$ is added to the tissue conductivity values according to

$$
\sigma_{\text {noise }}=\sigma+\delta \operatorname{rand}()
$$

where $\sigma$ is the tissue conductivity and $\operatorname{rand}()$ is a random Gaussian noise with zero mean and variance one. Moreover, in real cases the electrode positions could be shifted approximately $1 \mathrm{~cm}$. Here we added 7 levels of random noise to generate the electrode misplacements between $4 \mathrm{~mm}$ and $10 \mathrm{~mm}$ for a $3 D$ head-model, see Fig. 1a. For all three noise studies, each level of the noise is run 500 times to do statistical analysis and the mean of the results are calculated.

\subsection{Modeling Tissue Conductivity Anisotropy}

This section describes the modeling of realistic white matter (WM) conductivity anisotropy, for the generation of realistic anisotropic high-resolution volume conductor models of the head. Conductivity anisotropy, directionally dependent, with a ratio of about 1 to 9 (normal to parallel to fibers) has been measured for brain WM by Nicholson, ${ }^{11}$ however, a robust and non-invasive direct measurement seems to be challenging. Nevertheless, 


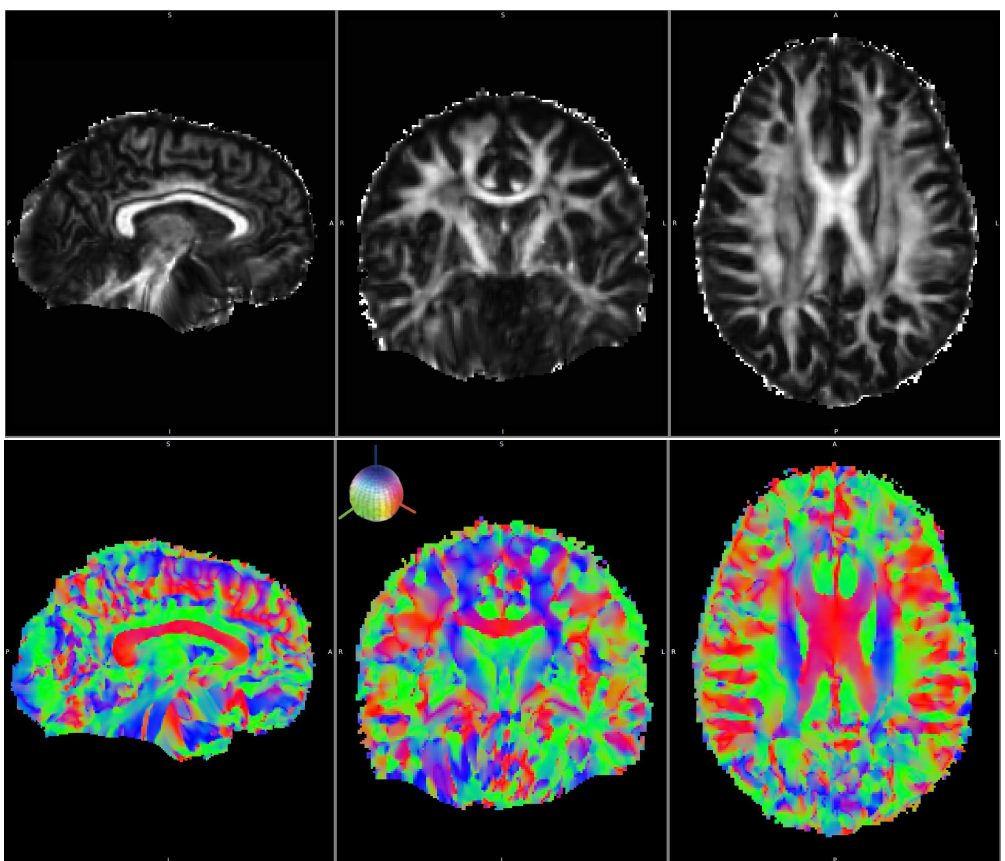

Figure 2: The fractional anisotropy image after registration of the T1 anatomy (top row), the color coded first eigenvector of the DTI tensor co-registered with the T1-weighted MRI (bottom row).

a formalism has been described recently for relating the effective electrical conductivity tensor to the effective water diffusion tensor in brain WM. ${ }^{12-14}$ Water diffusion can be measured non-invasively by DT-MRI. The mutual restriction of both the ionic and the water mobility by the geometry of the porous medium (the WM fibers) builds the basis for the described relationship. Basser et al. ${ }^{15}$ introduced the assumption that the conductivity tensor shares the eigenvectors with the water diffusion tensor. The assumption is not that a fundamental relation exists between the free mobility of ionic and water particles, rather that the restricted mobilities are related through the geometry.

Two approaches are proposed in the literature for extracting the tissue anisotropy from DT-MRI, namely a "direct mapping"16,17 and a "volume normalized mapping". ${ }^{18}$ The direct mapping simply scales the DTI tensors to get the conductivity distribution. The volume normalized mapping uses the anisotropy information of the DTI data, while maintaining the mean conductivity of the tensors at a predefined value, e.g., the WM or GM isotropic conductivity. This approach prevents the problem of very high peak conductivity values that can occur when the direct mapping is used.

The procedure to prepare the diffusion weighted images for the subsequent estimation of the conductivity tensors, is based on the processing steps implemented in FDT. ${ }^{19}$ A brain mask is extracted from the first bvalue, sensitivity to diffusion, equal to zero image and the remaining images are corrected for head movements and distortions caused by eddy-currents using a linear affine co-registration to this first b-value image. After fitting the diffusion tensors and determining the fractional anisotropy (FA), the FA image is co-registered to the structural T1-weighted image. A two-step procedure is used to account for local distortions in the diffusion weighted images, starting with an affine registration and then applying a nonlinear registration. The resulting warp field is applied to the DTI data, thereby ensuring that the correct diffusion directions are preserved. Fig. 2 shows the FA and color coded first eigenvector of the DTI tensor registered with the T1-weighted MRI, which illustrates the fiber orientation map from a DT-MRI. Finally, the conversion schemes from the diffusion to conductivity tensors are applied. In the next section we present two variations of these conversion schemes.

\subsubsection{Direct Mapping}

Tuch et al. in ${ }^{17}$ showed a linear relationship between the eigenvalues of the diffusion and conductivity tensors

$$
\sigma_{v}=s d_{v},
$$


where $\sigma_{v}$ and $d_{v}$ represent the $v$ th conductivity and diffusion eigenvalue, respectively, and $s$ is a scaling factor. With this assumption, the anisotropy ratio between the different diffusion eigenvalues are preserved. Tuch et al. ${ }^{17}$ in the original scaling factor reported that results often have unrealistically high conductivity values, and an adjusting scaling factor was applied to make sure that the conductivity stays in a reasonable range. ${ }^{16}$ The factor $s$ was selected such that the geometric mean of the conductivity eigenvalues, averaged across voxels, fitted that of the isotropic conductivities reported in the literature. Thereby, a single factor $s$ was chosen for GM and WM such that the mean conductivities derived from DTI for both tissue types matched the isotropic reference values as good as possible in a least-squares sense,

$$
s=\frac{\bar{d}_{W M} \sigma_{\mathrm{WM}}^{\mathrm{iso}}+\bar{d}_{\mathrm{GM}} \sigma_{\mathrm{GM}}^{\mathrm{iso}}}{\bar{d}_{\mathrm{WM}}^{2}+\bar{d}_{\mathrm{GM}}^{2}},
$$

where $\sigma_{\mathrm{WM}}^{\text {iso }}$ and $\sigma_{\mathrm{GM}}^{\text {iso }}$ denote the isotropic conductivities of WM and GM, respectively. Typical conductivities $\sigma_{\mathrm{WM}}^{\text {iso }}=0.142 \mathrm{~S} / \mathrm{m}$ and $\sigma_{\mathrm{GM}}^{\text {iso }}=0.33 \mathrm{~S} / \mathrm{m}$ were used as isotropic reference values for EEG source localization. ${ }^{16,20,21}$ In (4), the average value across voxels of the diffusion eigenvalues $d_{1}, d_{2}$ and $d_{3}$ is given by

$$
\bar{d}_{\mathrm{WM} / \mathrm{GM}}=\sqrt[3]{\frac{\sum_{k=1}^{N_{\mathrm{WM} / \mathrm{GM}}}\left(d_{1} d_{2} d_{3}\right)_{k}}{N_{\mathrm{WM} / \mathrm{GM}}}},
$$

where $N_{\mathrm{WM} / \mathrm{GM}}$ indicates the number of voxels corresponding to WM and GM, respectively.

\subsubsection{Volume Normalized Mapping}

An alternative for conductivity mapping from DTI is to locally match the geometric mean of the conductivity eigenvalues of each single voxel to that of an isotropic reference value. ${ }^{18}$ This approach is referred to as a "volume normalized" approach, with the adjusted conductivity eigenvalues being determined by

$$
\sigma_{i}=\frac{d_{i}}{\sqrt[3]{d_{1} d_{2} d_{3}}} \sigma_{\mathrm{WM} / \mathrm{GM}}^{\mathrm{iso}}
$$

\section{NUMERICAL RESULTS}

\subsection{Head-Model and Noise study}

For our simulations, virtual population head models were used, which consists of eight highly detailed anatomical whole-body models of adults and children. ${ }^{8}$ The models or numerical phantoms are based on high-resolution MR images of healthy volunteers. We chose the 11 year-old girl model. For the $2 D$ case a thin layer of the brain with $1 \mathrm{~mm}$ resolution was selected which had 10 tissues, see Fig. 1a. Thirty equally distant EEG electrodes were located around the model to measure the potential values. In the 3D case the head model had $2 \mathrm{~mm}$ resolution with five tissues (skull, skin, WM, GM and CSF), see Fig. 1b. The 61 EEG electrodes were placed on the head surface based on the 10/10 EEG electrode system ${ }^{9}$ and the conductivity values were taken from a database. ${ }^{10}$

To evaluate the results, three parameters were calculated; localization error (LE), relative error (RE) and orientation error $(\mathrm{OE})$. The LE is the distance between the estimated dipole position and the actual dipole position,

$$
L E=\left\|\mathbf{x}_{0}-\mathbf{x}_{\mathrm{est}}\right\|,
$$

where $\mathbf{x}_{0}$ and $\mathbf{x}_{\mathrm{est}}$ are actual and estimated dipole positions, respectively, and $\|\cdot\|$ is the Euclidean norm. The relative error is defined as:

$$
R E=\frac{\left\|\mathbf{u}_{\text {elec }}-\mathbf{u}_{\text {est }}\right\|}{\left\|\mathbf{u}_{\text {elec }}\right\|},
$$

where $\mathbf{u}_{\text {elec }}$ and $\mathbf{u}_{\text {est }}$ are actual and estimated electrode potentials, respectively. Moreover, the orientation error is defined as:

$$
O E=\cos ^{-1}\left(\frac{\mathbf{M}_{0} \cdot \mathbf{M}_{\mathrm{est}}}{\left\|\mathbf{M}_{0}\right\| \| \mathbf{M}_{\mathrm{est} \|}}\right) .
$$


Table 2: Numerical results for the head-model study.

\begin{tabular}{|c|c|c|c|c|c|c|}
\hline & Model\#1 & Model\#2 & Model\#3 & Model\#4 & Model\#5 & Model\#6 \\
\hline LE (mm) & 3 & 20 & 1 & 21 & 24 & 26 \\
\hline RE (\%) & 21.13 & 54.97 & 0.34 & 24.77 & 28.3 & 36.78 \\
\hline OE (deg) & 10 & 7.5 & 0.7 & 9.5 & 14 & 21.5 \\
\hline
\end{tabular}

where $\mathbf{M}_{0}$ and $\mathbf{M}_{\text {est }}$ are actual and estimated dipole moments, respectively.

Table 2 shows the results for the head-model study. As we can see from Table 2, Model 3 (includes skin, skull, WM, GM and CSF) had the smallest errors compared to the other models. Moreover, by analyzing the results with respect to the different tissues in the models, we can see that the models without the CSF had larger error compared to the others.

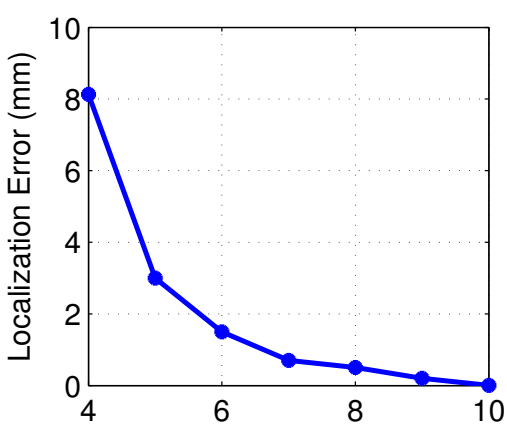

(a) SNR

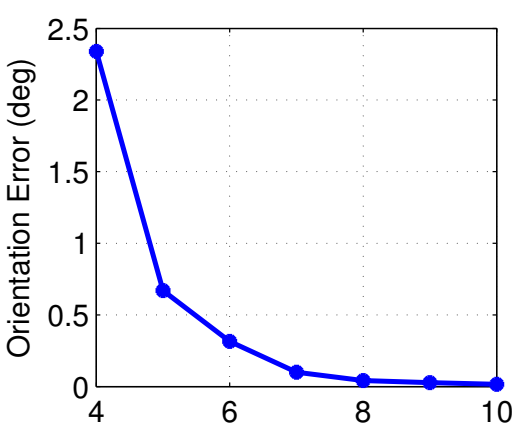

(b) SNR

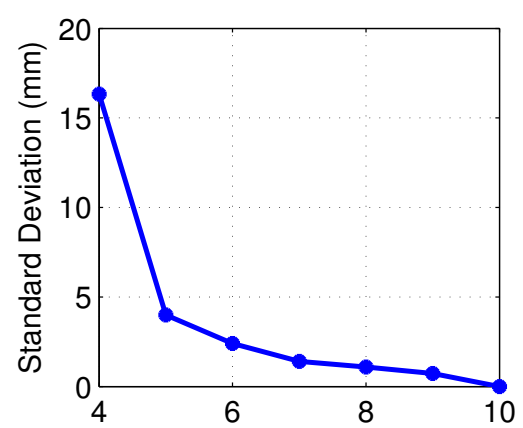

(c) SNR

Figure 3: (a) Localization error, (b) orientation error and (c) localization STD for Model 3 with noise added to the EEG signals. (2D case)
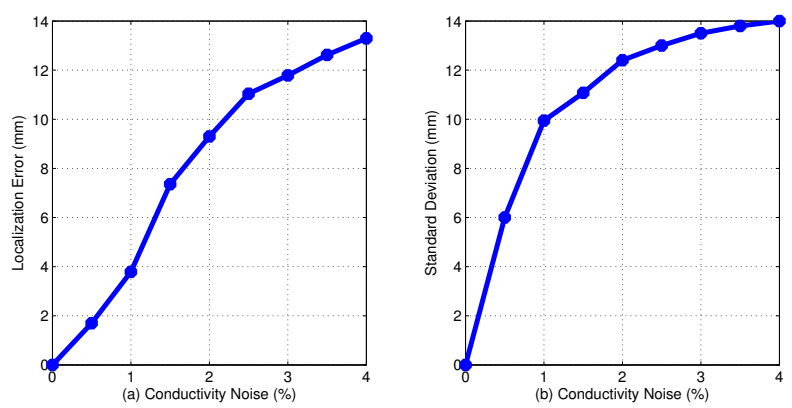

Figure 4: (a) Localization error and (b) its STD for Model 3 with noise added to the tissue conductivity values. (2D case)
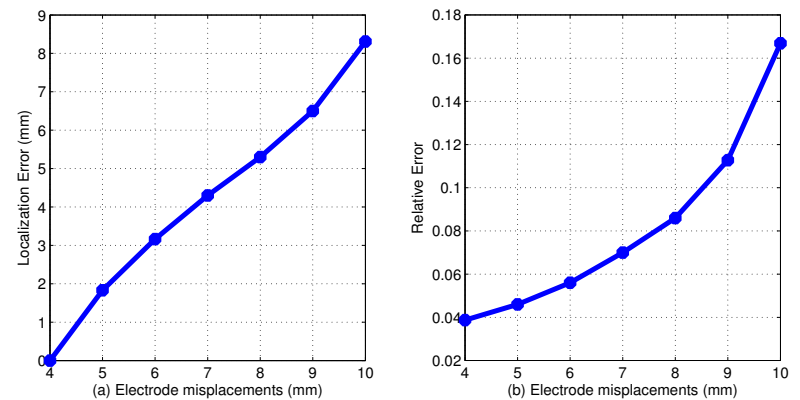

Figure 5: (a) Localization and (b) relative error for Model 3 with noise added to the electrode positions. (3D case)

Based on the result presented in Table 1, we used Model 3 for the noise study. In real EEG the background signals have a frequency between 8 to $13 \mathrm{~Hz}$, known as Alpha rhythm, and its amplitude is in the range 30 to $40 \mu \mathrm{V}$. The spike signals usually have duration in the range 30 to $70 \mathrm{~ms}$. The amplitude of these signals could be from 100 to $200 \mu \mathrm{V}$. We have added noise with different levels of SNR with respect to the background EEG. Fig. 3 shows results for EEG signals contaminated with noises. As shown in Fig. 3, the localization error is less than $2 \mathrm{~mm}$ for SNR equal to 6 and higher. Fig. 4 shows the results for Model 3 with conductivity noise. For conductivity noise equal to $4 \%$ the mean LE is approximately $13.6 \mathrm{~mm}$. Fig. 5 shows the results for electrode 


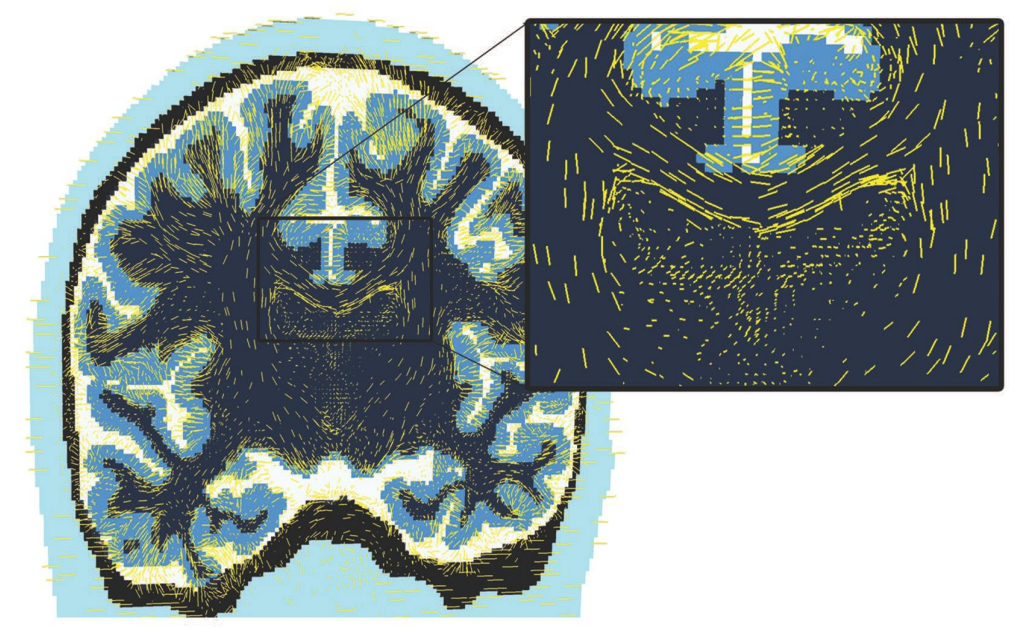

Figure 6: The maximum eigenvector co-registered to the segmented T1-weighted image. The zoom-in window shows the corpus callosum area which has the highly anisotropic structures.

position misplacements. For electrode misplacement around $10 \mathrm{~mm}$ the LE is approximately $8 \mathrm{~mm}$ and the RE is $17 \%$.

\subsection{White Matter Anisotropy}

Both the direct and the volume normalized mapping were tested for synthetic EEG source localization with a realistic head model to investigate the influences of anisotropic WM on EEG source localization. Fig. 6 illustrates the maximum eigenvector co-registered to the segmented T1-weighted image. The zoom-in window in Fig. 6 shows the corpus callosum area. The corpus callosum is the major white-matter tract that crosses the interhemispheric fissure in the human brain and it consists of approximately 200 million interhemispheric fibers, most of which connect homologous regions of the cerebral cortex. ${ }^{22}$ The corpus callosum with highly anisotropic cellular structures is a good reference to check the co-registration results visually.

To investigate the influence of WM anisotropy on source localization, a patch of gray matter with 725 voxels was selected. First, the EEG signals for a model with anisotropic tissues were calculated synthetically by solving the forward problem. Then the exhaustive search algorithm was used to locate the sources for a model with isotropic tissues. At each point, dipoles with three polarities, i.e., $\mathrm{x}-, \mathrm{y}$ - and z-polarity were tested. The relative errors (RE) are calculated by comparing the isotropic and anisotropic solution at each electrode node as follows

$$
R E=\frac{\left\|\mathbf{u}_{\text {elec }}^{\text {aniso }}-\mathbf{u}_{\text {elec }}^{\text {iso }}\right\|}{\left\|\mathbf{u}_{\text {elec }}^{\text {aniso }}\right\|},
$$

where $\mathbf{u}_{\text {elec }}^{\text {iso }}$ and $\mathbf{u}_{\text {elec }}^{\text {aniso }}$ are potential values at electrodes for isotropic and anisotropic models, respectively. Moreover, for a single point source, the localization error (LE) is the distance between the estimated and the actual

Table 3: Mean and standard deviation of the localization and relative error for 725 source positions with three polarities. The direct and the volume normalized (VN) mapping were used to calculate the WM anisotropic conductivities from diffusion tensor images.

\begin{tabular}{|c|ccc|ccc|}
\hline & \multicolumn{3}{|c|}{ Direct Mapping } & \multicolumn{3}{c|}{ VN Mapping } \\
\cline { 2 - 7 } & x-polarity & y-polarity & z-polarity & x-polarity & y-polarity & z-polarity \\
\hline LE $(\mathrm{mm})$ & $5.2 \pm 3.1$ & $6.1 \pm 3.5$ & $6.5 \pm 4.2$ & $4.1 \pm 2.2$ & $5.2 \pm 2.8$ & $5.7 \pm 3.4$ \\
\hline RE & $0.22 \pm 0.03$ & $0.26 \pm 0.04$ & $0.20 \pm 0.03$ & $0.19 \pm 0.04$ & $0.22 \pm 0.04$ & $0.17 \pm 0.05$ \\
\hline
\end{tabular}



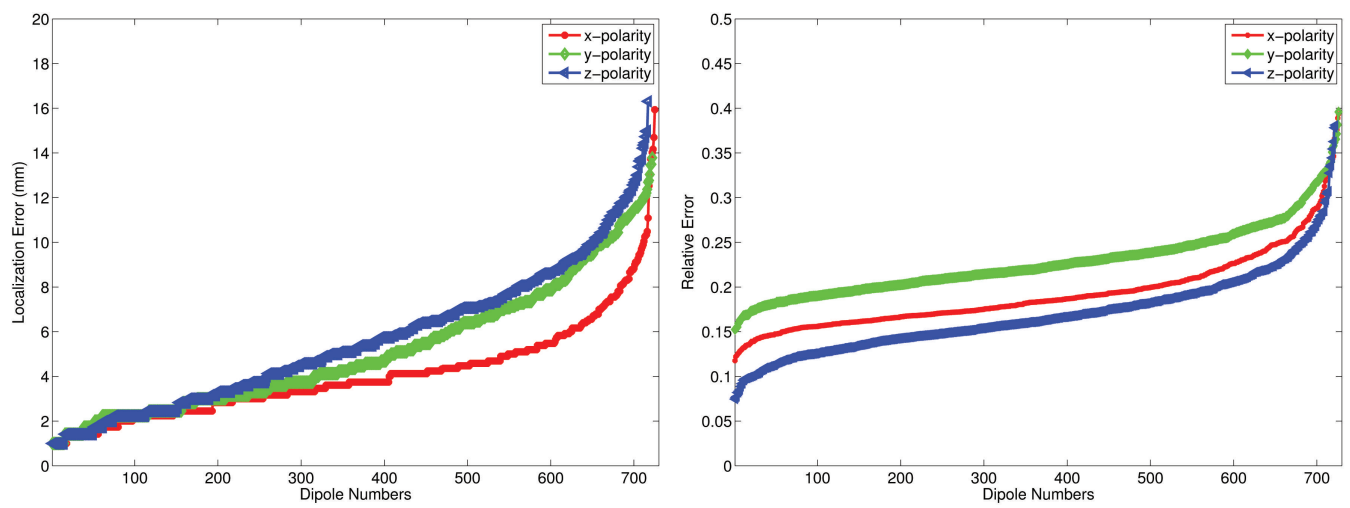

Figure 7: The localization error (left) and the relative error (right) for the different dipole sources using the volume normalized mapping.

source position, defined as

$$
L E=\left\|\mathrm{x}_{0}^{\text {aniso }}-\mathrm{x}_{0}^{\text {iso }}\right\|,
$$

where $\mathbf{x}_{0}^{\text {aniso }}$ is the actual source position in the anisotropic head model and $\mathbf{x}_{0}^{\text {iso }}$ is the estimated source position in the isotropic head model.

Table 3 presents the mean and standard deviation (STD) of the LE and RE for both direct and volume normalized mapping. As we can see the WM anisotropy affects the localization approximately $5 \mathrm{~mm}$ with a $20 \%$ relative error. Fig. 7 shows the localization and relative error results for all three polarities. As we can see in Fig. 7 the x-polarity dipoles have smaller localization error compared to other polarities. Fig. 8 shows the relative error projected on the gray matter voxels. This figure indicates that the source positions with strong support, surrounded by gray matter voxels, have smaller relative compared to those which are closer to or on the boundary.

\section{CONCLUSIONS AND DISCUSSION}

In this paper we studied the influence of head-model complexity and different sources of noise on the EEG source localization. Six finite element head models constructed from MR images were selected for a head-model complexity study. A reference head model was used to create synthetic EEG signals by placing a dipole source inside the model to mimic an epileptic spike activity. For the inverse problem an exhaustive search method was used to estimate the best dipole position and orientation. To investigate the influence of white matter anisotropy a realistic head model generated form T1-weighted images was used, the conductivity anisotropy for white matter was extracted from the diffusion tensor imaging (DTI) data. Results showed that the inverse problem is significantly influenced by the head-model complexity. Based on the model study presented here, the inverse problem had large localization error for models without the CSF. As normal CSF has long T1 and long T2 times that manifest as dark signal on T1-weighted images and bright signal on T2-weighted images it is difficult to segment the CSF accurately. Moreover, the brain extraction step during brain segmentation can affect the CSF miss-classification significantly since a large amount of CSF is placed in between the brain and skull compartments. ${ }^{23}$ For the accurate EEG source localization it is very important to be aware of the CSF miss-classification. The noise study results showed that the EEG source localization is very sensitive to the tissue conductivity errors and only $4 \%$ noise on conductivity can cause $13 \mathrm{~mm}$ localization error. Therefore, an accurate source localization requires accurate knowledge of tissue conductivity, thus improved measurement methods of tissue properties are necessary. This is in agreement with other publications. ${ }^{24}$ Electrode misplacements can make approximately $17 \% \mathrm{RE}$ and $8 \mathrm{~mm} \mathrm{LE}$ for $1 \mathrm{~cm}$ electrode positioning error. Although, most of the EEG electrode digitizers used in a clinical measurement routing have measurement accuracy around $1 \mathrm{~mm}$, patient movements may shift the EEG electrode positions during a recording. To avoid electrode misplacement error there is a strong need to develop an electrode positioning system which can localize the electrode positions over time during a session. ${ }^{25}$ 


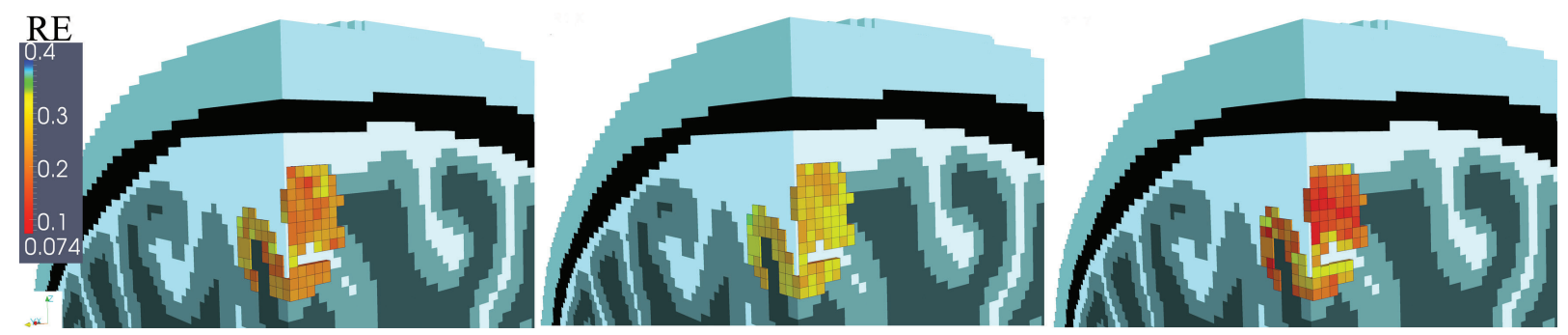

Figure 8: The color coded relative error projected on the gray matter for dipoles with x-polarity (left), y-polarity (middle) and z-polarity (right) obtained using the volume normalized mapping.

From the white matter anisotropy investigation, we concluded that using a head model with white matter anisotropic tissue might affect the source localization in the range of millimeters. These results are consistent with a previous study, ${ }^{18}$ which concluded that the single-source localization errors resulting from neglecting anisotropy were found to be smaller compared to errors associated with other modeling errors, such as misclassified tissue or the use of nonrealistic head models.

\section{REFERENCES}

1. De Munck J. C., "The potential distribution in a layered anisotropic spheroidal volume conductor". Journal of Applied Physics, 64(2):464-470, 1988.

2. van den Broeh, S., Zhou, H., and Peters, M. "Computation of neuromagnetic fields using finite-element method and Biot-Savart law". Medical and Biological Engineering and Computing, 34(1):21-26, 1996.

3. Edelvik, F., Andersson, B., Jakobsson, S., Larsson, S., Persson, M., and Shirvany, Y. "An improved method for dipole modeling in EEG-based source localization". In World Congress on Medical Physics and Biomedical Engineering, volume 25 of IFMBE Proceedings, pages 146-149. 2009.

4. Hallez, H., Vanrumste, B., Grech, R., Muscat, J., De Clercq, W., Vergult, A., D'Asseler, Y., Camilleri, K. P., Fabri, S. G., Van Huffel, S., and Lemahieu, I. "Review on solving the forward problem in EEG source analysis". Neuroengineering and Rehabilitation, 46(4), 2007.

5. Shirvany, Y., Edelvik, F., Jakobsson, S., Hedström, A., and Persson, M. "Application of particle swarm optimization in epileptic spike EEG source localization". Applied Soft Computing, in press, Available online, 2013.

6. Wang, Y. and Gotman, J. "The influence of electrode location errors on EEG dipole source localization with a realistic head model". Clinical Neurophysiology, 112(9):1777 - 1780, 2001.

7. Yeung, N., Bogacz, R., Holroyd, C. B., and Cohen, J. D. "Detection of synchronized oscillations in the electroencephalogram: An evaluation of methods". Psychophysiology, 41(6):822-832, 2004.

8. http://www.itis.ethz.ch/services/human-and-animal-models/human-models/.

9. Jurcak, V., Tsuzuki, D., and Dan, I. "10/20, 10/10, and 10/5 systems revisited: Their validity as relative head-surface-based positioning systems". NeuroImage, 34(4):1600 - 1611, 2007.

10. http://niremf.ifac.cnr.it/tissprop/.

11. Nicholson, P. W. "Specific impedance of cerebral white matter". Experimental Neurology, 13(4):386 - 401, 1965.

12. Tuch, D. S., Wedeen, V. J., Dale, A. M., and Belliveau, J. W. "Electrical conductivity tensor map of the human brain using NMR diffusion imaging: An effective medium approach". ISMRM, 6th Scientific Meeting, Sydney, 1998.

13. Tuch, D. A. S., Wedeen, van J., Dale, A. M., George, J. S., and Belliveau, J. W. "Conductivity mapping of biological tissue using diffusion MRI". Annals of the New York Academy of Sciences, 888(1):314-316, 1999.

14. Sekino, M., Inoue, Y., and Ueno, S. "Magnetic resonance imaging of electrical conductivity in the human brain". Magnetics, IEEE Transactions on, 41(10):4203 - 4205, 2005.

15. Basser, P.J. ,Mattiello, J., and LeBihan, D. "MR diffusion tensor spectroscopy and imaging". Biophysical Journal, 66(1):259 - 267, 1994. 
16. Rullmann, M., Anwander, A., Dannhauer, M., Warfield, S. K., Duffy, F. H., and Wolters, C. H. "EEG source analysis of epileptiform activity using a $1 \mathrm{~mm}$ anisotropic hexahedra finite element head model". Neuroimage, 44:399-410, 2009.

17. Tuch, D. S., Wedeen, V. J., Dale, A. M, George, J. S., and Belliveau, J. W. "Conductivity tensor mapping of the human brain using diffusion tensor MRI". Proc Natl Acad Sci USA, 98:11697-11701, 2001.

18. Güllmar, D., Haueisen, J., and Reichenbach, J. R. "Influence of anisotropic electrical conductivity in white matter tissue on the EEG/MEG forward and inverse solution. A high-resolution whole head simulation study". Neuroimage, 51:145-163, 2010.

19. Smith, S. M. ,Jenkinson, M., and et al. "Advances in functional and structural MR image analysis and implementation as FSL". NeuroImage, 23:208-219, 2004.

20. Gabriel, S., Lau, R.W., and Gabriel, C. "The dielectric properties of biological tissues: III parametric models for the dielectric spectrum of tissues". Phys Med Biol, 41(11):2271 - 2293, 1996.

21. Haueisen, J., Ramon, C. and Schimpf, P. H. "Influence of head models on EEG simulations and inverse source localizations". Biomed Eng., page online 5, 2006.

22. Biegon, A., Eberling, J. L., Richardson, B. C., and et al. "Human corpus callosum in aging and alzheimer's disease: a magnetic resonance imaging study". Neurobiol Aging, 15:393-397, 1994.

23. Shirvany, Y., Porras, A. R., Kowkabzadeh, K., Mahmood, Q., Lui, H. S., and Persson, M. "Investigation of brain tissue segmentation error and its effect on EEG source localization". In Engineering in Medicine and Biology Society (EMBC), 2012 Annual International Conference of the IEEE, pages 28(1):1522-1525, 2012.

24. Shen, I., Johnson, G., Gohra, H., Bardy, G. H., Haynor, D. R., Jorgenson, D. B., Schimpf, P. H. and Kim, Y. "Predicting cardiothoracic voltages during high-energy shocks: Methodology and comparison of experimental to finite element model data". IEEE Trans. Biomed. Eng., 42(6):559-571, 1995.

25. Shuo, Q. and Yang, S. "A single camera photogrammetry system for multi-angle fast localization of EEG electrodes". Annals of Biomedical Engineering, 39(22):2844-2856, 2011. 